\title{
How many explosions does one need? - Quantifying supernovae in globular clusters from iron abundance spreads
}

\author{
Henriette Wirth ${ }^{1,2 \star}$, Tereza Jerabkova ${ }^{3}$, Zhiqiang Yan $^{2,4}$, Pavel Kroupa ${ }^{2,4}$, \\ Jaroslav $\mathrm{Haas}^{2}$ and Ladislav $\breve{S} u b^{2}$ \\ ${ }^{1}$ Elektronische Fahrwerksysteme GmbH, Dr.-Ludwig-Kraus-Str. 6, 85080 Gaimersheim, Germany \\ ${ }^{2}$ Charles University, Faculty of Mathematics and Physics, Astronomical Institute, V Holešovičkách 2, CZ-18000 Praha 8, Czech Republic \\ ${ }^{3}$ European Space Agency (ESA), European Space Research and Technology Centre (ESTEC), Keplerlaan 1, 2201 AZ Noordwijk, \\ The Netherlands \\ ${ }^{4}$ Helmholtz Institut für Strahlen und Kernphysik, Universität Bonn, Nussallee 1416, 53115 Bonn, Germany
}

Accepted XXX. Received YYY; in original form ZZZ

\section{ABSTRACT}

Many globular clusters (GCs) are known to host multiple populations distinguishable by their light-element content. Less common are GCs displaying iron abundance spreads which are seen as evidence for enrichment through core collapse supernovae (SNe). We present a simple analytical method to estimate the number of SNe required to have occurred in a GC from its metallicity and iron abundance spread. We then use this result to estimate how long star formation (SF) lasted to build the GC. We apply our method to up-to-date measurements and find that, assuming the correctness of these measurements, multiple SNe (up to $10^{5}$ ) are required in most GCs with iron abundance spreads. The number of SNe events which contributed to the enrichment of the GCs studied here is typically a factor of 10 less than the expected number of SNe in a canonical initial mass function (IMF). This indicates that gas expulsion from the forming GC occurred after the first 10 per cent of SNe exploded. We compute that for the GCs typically SF ends after only a few Myr (extending up to $\approx 30 \mathrm{Myr}$ in a few cases). We also discuss possible improvements of this method and especially its sensitivity to the error of iron abundance measurements of individual stars of a GC. The method presented here can quickly give an estimate for the number of SNe required to explain the iron abundance spread in a GC without the requirement of any hydrodynamical simulations.

Key words: globular clusters: general - supernovae: general - stars: abundances - methods: analytical

\section{INTRODUCTION}

Many globular clusters (GCs) are known to host multiple populations of stars. These can be distinguished by their different abundances of the light elements $\mathrm{He}, \mathrm{C}, \mathrm{N}, \mathrm{O}, \mathrm{Na}, \mathrm{Al}$. The origins of the differences in individual GCs remain unclear (see e.g. Gratton et al. 2012, 2019; Wang et al. 2020, and references therein). One of the most common tools to detecting multiple populations are chromosome maps, that are constructed from two-colour diagrams using well-selected filters and combinations thereof together with spectroscopy allowing to efficiently distinguish populations with different light-element abundances (Milone et al. 2015; Milone 2016; Milone et al. 2017). While most GCs are considered to be homogeneous as far as their iron content is concerned (Carretta et al. 2009b; Mucciarelli et al. 2015; Montecinos et al. 2021), strong evidence for iron abundance spreads has been found in some of them (Pancino et al. 2000; Ferraro et al. 2009; Lardo et al. 2016; Marino et al. 2018). In the sample

* E-mail: henri-ette_w@web.de (HW) used in this work the GCs with large iron spreads (likely not to be a consequence of measurement errors) are: NGC 5139, NGC 5272, NGC 5286, NGC 5634, NGC 6205, NGC 6273, NGC 6341, NGC 6656, NGC 6715, Terzan 5 and Terzan 8. The question then becomes: Where do these iron abundance spreads come from?

If we assume that the GCs initially formed out of a homogeneous gas cloud, no iron abundance spreads would be expected in the stars that formed prior to the first core collapse supernovae (SNe). The most massive stars in the cluster evolve and produce iron before they finally die in a core collapse SN (Woosley \& Weaver 1995; Portinari et al. 1998). Through this SN a small portion of the iron from the star is released (Woosley \& Weaver 1995; Portinari et al. 1998; Maoz \& Graur 2017), which then mixes with any still existing surrounding gas. If star formation (SF) has not ceased at this point, the now iron enriched gas collapses and forms stars with a slightly higher iron content than the previous one. This would lead to the observed iron abundance spreads, next to spreads of other elements (Umeda \& Nomoto 2003). Jiménez et al. (2021) estimated the iron spreads expected 
for different initial conditions. However, we could also use the knowledge about the observed iron spreads to estimate the number of SNe that must have exploded This is especially relevant, since $\mathrm{SNe}$ are seen as responsible for ending SF by blowing out the remaining gas from the GC (Shustov \& Wiebe 2000; Baumgardt et al. 2008; Krause et al. 2012). Therefore, we can use this to better understand when SF in GCs ended. We implicitly assume here that the GCs formed their stars in subsequent star formation periods. However, we are not following the AGB scenario proposed by Cottrell \& Da Costa (1981), in which the second generation forms only after the SNe have expelled all the gas. In the AGB scenario, the gas the second generation forms out of comes from the ejecta of AGB stars and accreted primordial gas. While the AGB scenario is likely to contribute to the multiple populations problem in GCs, the process studied here is more similar to the extended SF scenario proposed by Elmegreen (2017). Here the star formation continues, while the stars in the dense young GC lose gas through stellar evolution and mergers. Particularly important in this context is that discrete subpopulations of stars with different light-element abundances may arise within the so forming GC through mergers of massive stars for which the initial binary fraction is expected to be very high (Wang et al. 2020). This pollutes the gas in the $\mathrm{GC}$ from which enriched stars form. The GC formation process studied here allows for SF to continue after the first SNe. It is yet to be investigated if this is a viable scenario able to explain all chemical abundances in GCs.

The purpose of this paper is to present a method to estimate the number of SNe required to produce the observed iron abundance spreads. In Sec. 2 we describe the mathematical methods used in this work. We apply our method to the data given in Bailin (2019) in Sec. 3 and discuss how the model could be improved in Sec. 4. We summarise our findings in Sec. 5.

\section{METHODS}

\subsection{Calculating the number of SNe required}

In the scenario studied here the GC initially forms from one homogeneous gas cloud i.e. the iron abundance is the same for all stars. The most massive stars turn into SNe and pollute the gas that remained in the cluster with iron. If stars form from this gas, stars with different iron abundances appear in one GC. An observed iron spread implies that SF must have been going on after the first $\mathrm{SNe}$ exploded, which is at least for $3 \mathrm{Myr}$, but may be longer depending on the upper mass limit of the GC stars (Ekström et al. 2012). For our calculations we assume that the GC does not loose any gas or stars before the iron enriched generation has formed and that the gas is well mixed, i.e. the polluted gas becomes homogeneous before new stars form.

To calculate the number of $\mathrm{SNe}$ required to reproduce the iron abundance spreads observed in the GCs we first compute the total amount of iron that needs to be created. We assume that the initial metallicity of the GCs was $\left([\mathrm{Fe} / \mathrm{H}]-\sigma_{[\mathrm{Fe} / \mathrm{H}]}\right)$ and after the gas was enriched by SNe the metallicity of the remaining gas was $\left([\mathrm{Fe} / \mathrm{H}]+\sigma_{[\mathrm{Fe} / \mathrm{H}]}\right)$, where $[\mathrm{Fe} / \mathrm{H}]$ is the mean iron abundance of the GC and $\sigma_{[\mathrm{Fe} / \mathrm{H}]}$ the width of the Gaussian fitted over the iron distribution as given in Bailin
(2019). For more information on the data used see Sec. 2.3. We use twice the Gaussian width as our spread, since we assume the metallicity of the first generation to be more towards the lower end of the distribution, which would make the spread larger. This assumes that the mean metallicity of the GC is exactly in the middle of the metallicities of the two generations. For a GC with a pre-SNe-enriched generation that is larger than the post-SNe-enriched generation the average metallicity of the GC would be lower and since we are using a logarithmic scale, we would underestimate the amount of iron required and vice versa. This could be improved using detailed histograms showing the metallicities of the stars of the GC to determine the metallicities of the individual generations.

Additionally, we neglect any gas loss and assume that the gas is well mixed. The mass of iron in $M_{\odot}$ contained in a star of $1 M_{\odot}$ with metallicity $[\mathrm{Fe} / \mathrm{H}]$ is:

$m_{\text {iron,star }}=p_{\text {iron, } \odot} 10^{[\mathrm{Fe} / \mathrm{H}]}$,

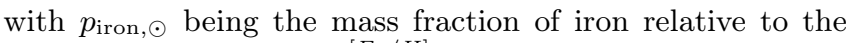
total mass of the Sun. $10^{[\mathrm{Fe} / \mathrm{H}]}$ produces the mass fraction of iron with respect to hydrogen over that of the Sun. However, the hydrogen fraction in the GC stars are not necessarily the same. Therefore an additional factor would be needed to account for this discrepancy. For our estimate, however, we assume that all stars have the same hydrogen content and set this factor to 1 . Note that the mass fraction of iron with respect to hydrogen, $[\mathrm{Fe} / \mathrm{H}]$, equals the number fraction usually measured. $10^{\left(\log \left(M_{\mathrm{Fe}, \mathrm{star}} / M_{\mathrm{H}, \mathrm{star}}\right)-\log \left(M_{\mathrm{Fe}, \odot} / M_{H, \odot}\right)\right)}=$ $\left(N_{\mathrm{Fe}, \text { star }} / N_{H, \text { star }}\right) /\left(N_{\mathrm{Fe}, \odot} / N_{H, \odot}\right)=10^{[\mathrm{Fe} / \mathrm{H}]}$ since the atomic masses cancel out.

To compute the total mass of iron needed we simply substract the iron in $1 M_{\odot}$ of the first generation from that of $1 M_{\odot}$ of the second generation and scale the difference up to the total mass of the gas left over in the GC:

$$
\begin{aligned}
M_{\text {iron }}= & p_{\text {iron }, \odot}\left(10^{[\mathrm{Fe} / \mathrm{H}]+\sigma_{[\mathrm{Fe} / \mathrm{H}]}}-10^{[\mathrm{Fe} / \mathrm{H}]-\sigma_{[\mathrm{Fe} / \mathrm{H}]}}\right) \\
& \times M_{\mathrm{ecl}}\left(\frac{1}{\epsilon}-1\right),
\end{aligned}
$$

with $M_{\text {iron }}$ being the total amount of iron to be created by the SNe in a GC to account for its stellar iron spread, $M_{\mathrm{ecl}}$ the initial mass of the stars in the still gas-embedded GC and the SF efficiency is $\epsilon=\frac{M_{\text {ecl }}}{M_{\text {gas }}}$, where $M_{\text {gas }}$ is the gas mass of the molecular cloud core in which the GC forms. In this work we assume $\epsilon=0.3$ (see e.g. Lada \& Lada 2003; André et al. 2014; Megeath et al. 2016; Banerjee \& Kroupa 2018) and $p_{\text {iron, } \odot}=0.0013$ calculated from the measurements given in Asplund et al. (2009). This equation can be rewritten an:

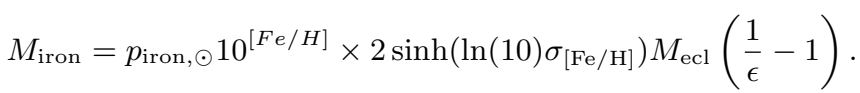

$\sinh (x) \approx x$ for small values of $x$. This means that we expect for $M_{\text {iron }}$ and as we will see the number of SNe to scale linearly with $\sigma_{[\mathrm{Fe} / \mathrm{H}]}$ as long as $\sigma_{[\mathrm{Fe} / \mathrm{H}]}$ remains small.

Taking the iron yield for a core collapse SN to be $0.074 M_{\odot}$ (Maoz \& Graur 2017) and dividing the total amount of iron through this value gives an estimate of the number of $\mathrm{SNe}$ required:

$N_{\mathrm{SN}}=\frac{M_{\text {iron }}}{0.074 M_{\odot}}$. 
We used the values for $[\mathrm{Fe} / \mathrm{H}]$ and $\sigma_{[\mathrm{Fe} / \mathrm{H}]}$ from Bailin (2019) in this work.

Based on the gas expulsion computations by Brinkmann et al. (2017) we assume that the GC looses a negligible fraction of its stars when its residual gas is expelled, i.e. $M_{\mathrm{ini}}=M_{\mathrm{ecl}}$ to a good approximation. To calculate $M_{\mathrm{ini}}$ for each GC in our sample we use Eq. 10 from Baumgardt \& Makino (2003), which describes the dissolution time of the GC depending on the initial number of stars, and their Eq. 12, which describes how the GC mass changes over time. These give us:

$0=\beta\left[\frac{\frac{M_{\mathrm{ini}}}{\langle m\rangle}}{\ln \left(\gamma \frac{M_{\mathrm{ini}}}{\langle m\rangle}\right)}\right]^{x} \frac{R_{\mathrm{ap}}}{\mathrm{kpc}}(1-e) \frac{1-\frac{M(t)}{0.7 M_{\mathrm{ini}}}}{\frac{t}{\mathrm{Myr}}}-1$.

We assume constant parameters $\beta=1.91, x=0.75$, a Coulomb logarithm, $\gamma=0.02$, and a mean stellar mass of $\langle m\rangle=0.579 M_{\odot}$, which matches the mean stellar mass for the canonical initial mass function (IMF) (Kroupa 2001; Kroupa et al. 2013). $\beta=1.91, x=0.75$ are fitting parameters of the model from Baumgardt \& Makino (2003), which depend on the central concentration. We assumed a rotational velocity of the Galaxy of $220 \mathrm{~km} \mathrm{~s}^{-1}$ (Blitz 1979; Honma \& Sofue 1997). The values used here were found for GCs with a King concentration parameter of 5.0 on circular orbits. We took the current mass $M(t)$ and orbit parameters of the GCs from the GC catalog compiled by Hilker et al. (2019). $R_{\text {ap }}$ is the apocentre of the GC and $e$ its eccentricity, which we calculated using apo- and pericentre of the GC.

The GC age $t$ is assumed to be $12 \mathrm{Gyr}$ in accordance with literature values (Dotter et al. 2010; Usher et al. 2019). Dotter et al. (2010) looked at 60 Milky Way (MW) GCs. They derived their results by first measuring the magnitude of the main-sequence turnoff and applied isochromes depending on age and metallicity. This resulted in all but two of their GCs having ages between 10 and 14 Gyr (See their Table 2). Usher et al. (2019) fit photometric measurements with synthetic models for $83 \mathrm{MW}$ GCs. They derive GC ages between 5.5 and 14.5 Gyr. All the parameters are, therefore, estimated reasonably well and we solve this equation implicitly for $M_{\mathrm{ini}}$ for each GC using the Newton-Raphson method.

\subsection{Computing when star formation ends}

If we know how many SNe have to explode for the next generation to form, we can use this to estimate the time until $\mathrm{SF}$ ends. We assume, that gas mixing and SF happens instantaneously. This means that SF ends after the $N_{\mathrm{SN}}$ th SN took place and before the $\left(N_{\mathrm{SN}}+1\right)$ th happenes. We use the canonical IMF (Kroupa 2001; Kroupa et al. 2013) for stellar masses between $0.08 M_{\odot}$ and $m_{\max }=120 M_{\odot}$ to compute the number of SNe expected to happen. The minimum mass for a SN progenitor is assumed to be $m_{\mathrm{SN}}=8 M_{\odot}$. From this we can calculate that about $0.6 \%$ of the cluster stars are more massive than $8 M_{\odot}$ which means that they will turn into SNe. The possibility of failed SNe as discussed in Pejcha \& Thompson (2015); Sukhbold et al. (2016); Basinger et al. (2020); Neustadt et al. (2021) is neglected here (for more discussion see Sec. 4.1). Therefore, the expected number of SNe, $N_{\mathrm{SN}}^{\exp }$ is:

$N_{\mathrm{SN}}^{\exp }=0.006 \frac{M_{\mathrm{ini}}}{\langle m\rangle}=0.0109 \frac{M_{\mathrm{ini}}}{M_{\odot}}$.
We use the integral of the upper end of the IMF:

$N_{\mathrm{SN}}^{\exp }=\int_{m_{S N}}^{m_{\max }} d m k \xi(m)$,

where $k$ is obtained by normalising the IMF to $M_{\mathrm{ini}} \cdot \xi(m)=$ $m^{-\alpha_{i}}$ is the canonical IMF with $\alpha_{1}=1.3$ for $0.08 \leq m / M_{\odot}<$ 0.5 and $\alpha_{2}=2.3$ for $0.5 \leq m / M_{\odot} \leq 120$. With this we can compute the mass of the $N_{\mathrm{SN}}$ th SN, $m_{\text {last }}$, to explode:

$\frac{N_{\mathrm{SN}}}{N_{\mathrm{SN}}^{\exp }}=\frac{m_{\mathrm{max}}^{-1.3}-m_{\text {last }}^{-1.3}}{m_{\max }^{-1.3}-m_{\mathrm{SN}}^{-1.3}}$.

It is assumed here that the iron output of $\mathrm{SNe}$ is the same for all stars (i.e. no mass dependency). We use the correlation between $m_{\text {last }}$ and its lifespan shown in fig. 3 of Yan et al. (2019) for $[\mathrm{Fe} / \mathrm{H}]=-1.67$ to compute the time until SF ends. Their fig. 3 shows how the lifespan of stars decreases with increasing stellar mass. Most of our GCs have a low metallicity and the lifespans of stars above $8 M_{\odot}$ do not vary significantly at low metallicity.

\subsection{The dataset used}

The catalogue by Bailin (2019) can be seen as an extension of the catalogue from Willman \& Strader (2012). It is the largest catalogue of iron spreads in GCs to date. They combine several studies of red giant branch measurements in GCs. Combining different data sources like this produces an artificially inflated $\sigma_{[\mathrm{Fe} / \mathrm{H}]}$. Bailin (2019) used a Bayesian Markov Chain Monte Carlo method to correct for this.

They also argue that the error estimates in some of the studies they use are overestimated and correct for this. A discussion of the dataset and the impact of those errors is given in Sec. 4.1 and App. A.

\section{RESULTS}

In Table 1 we see the current and initial masses, metallicities $[\mathrm{Fe} / \mathrm{H}]$, iron abundance spreads $\sigma_{[\mathrm{Fe} / \mathrm{H}]}$, required number of SNe per unit mass, $n_{\mathrm{SN}}$, required numbers of $\mathrm{SNe}, N_{\mathrm{SN}}$, calculated for the iron abundance spreads given in Bailin (2019). The next columns show the number of SNe expected to occur in the GC given its initial mass, the estimated time at which SF ends and the number of SNe required corrected for the measurement error of metallicity measurements of individual stars. For the GCs in our sample, the number of SNe required to produce the observed iron abundance spreads is between five and a few tens of thousand with $\mathrm{SN}$ densities between $2.2 \times 10^{-5}$ and $7.4 \times 10^{-3} M_{\odot}^{-1}$. The exeption is Terzan 5 with a number of SNe per mass of $5.1 \times 10^{-2} M_{\odot}^{-1}$. This contradicts previous claims that only a few $\mathrm{SNe}$ can occur before the remaining gas is blown out of the GC and SF stops (Shustov \& Wiebe 2000).

Fig. 1 shows $n_{\mathrm{SN}}$ from Table 1 over the metallicities of the GCs. A positive dependency of $n_{\mathrm{SN}}$ on $[\mathrm{Fe} / \mathrm{H}]$ implied by our analytical model exists for the data in our sample. It is, however, somewhat weaker if we correct for the individual measurement errors for the metallicities of individual stars (see Fig. A1 and Sec. A). Contrary to theoretical predictions (Bailin 2018), we do not find any correlation between $n_{\mathrm{SN}}$ and $M_{\mathrm{ini}}$. 
Table 1. The current mass, $M(t)$, initial mass, $M_{\text {ini }}$, metallicity $[\mathrm{Fe} / H]$, iron abundance spread $\sigma_{[F e / H]}$, number of SNe per unit mass, $n_{\mathrm{SN}}=\frac{N_{\mathrm{SN}}}{M_{\mathrm{ini}}}$, number of SNe, $N_{\mathrm{SN}}$, the expected number of SNe to occur in the GC, $N_{\mathrm{SN}}^{\mathrm{exp}}$, the time SF ends, $t_{\mathrm{end}}^{\mathrm{SF}}$, and the number of $\mathrm{SNe}$ after correcting for the measurement error, $N_{\mathrm{SN}}^{\mathrm{subt}}$, calculated for our sample of GCs.

\begin{tabular}{|c|c|c|c|c|c|c|c|c|c|}
\hline Name & $M(t)$ & $M_{\mathrm{ini}}\left[10^{5} M_{\odot}\right]$ & {$[\mathrm{Fe} / \mathrm{H}]$} & $\sigma_{[\mathrm{Fe} / \mathrm{H}]}$ & $n_{\mathrm{SN}}\left[M_{\odot}^{-1}\right]$ & $N_{\mathrm{SN}}$ & $N_{\mathrm{SN}}^{\exp }$ & $t_{\text {end }}^{S F}[\mathrm{Myr}]$ & $N_{\mathrm{SN}}^{\mathrm{subt}}$ \\
\hline 47 Tuc & 8.07 & 12.85 & -0.747 & 0.033 & $1.18 \times 10^{-3}$ & $1.52 \times 10^{3}$ & $1.33 \times 10^{4}$ & 5.6 & $-6.27 \times 10^{2}$ \\
\hline NGC 288 & 0.98 & 2.88 & -1.226 & 0.037 & $4.39 \times 10^{-4}$ & $1.27 \times 10^{2}$ & $2.99 \times 10^{3}$ & 4.1 & $-3.32 \times 10^{1}$ \\
\hline NGC 362 & 3.36 & 8.69 & -1.213 & 0.074 & $9.09 \times 10^{-4}$ & $7.89 \times 10^{2}$ & $9.00 \times 10^{3}$ & 5.0 & $2.93 \times 10^{2}$ \\
\hline NGC 1851 & 2.81 & 8.48 & -1.157 & 0.046 & $6.41 \times 10^{-4}$ & $5.43 \times 10^{2}$ & $8.79 \times 10^{3}$ & 4.5 & $-7.74 \times 10^{0}$ \\
\hline NGC 1904 & 1.56 & 6.46 & -1.550 & 0.027 & $1.52 \times 10^{-4}$ & $9.81 \times 10^{1}$ & $6.69 \times 10^{3}$ & 3.6 & $-7.18 \times 10^{1}$ \\
\hline NGC 2419 & 14.30 & 20.79 & -2.095 & 0.032 & $5.14 \times 10^{-5}$ & $1.07 \times 10^{2}$ & $2.15 \times 10^{4}$ & 3.5 & $-4.94 \times 10^{1}$ \\
\hline NGC 2808 & 8.69 & 17.45 & -1.120 & 0.035 & $5.30 \times 10^{-4}$ & $9.25 \times 10^{2}$ & $1.81 \times 10^{4}$ & 4.2 & $-3.09 \times 10^{2}$ \\
\hline NGC 3201 & 1.41 & 2.41 & -1.496 & 0.044 & $2.81 \times 10^{-4}$ & $6.76 \times 10^{1}$ & $2.49 \times 10^{3}$ & 3.8 & $-4.15 \times 10^{0}$ \\
\hline NGC 4590 & 1.32 & 2.06 & -2.255 & 0.053 & $5.89 \times 10^{-5}$ & $1.21 \times 10^{1}$ & $2.13 \times 10^{3}$ & 3.5 & $1.43 \times 10^{0}$ \\
\hline NGC 4833 & 1.80 & 6.76 & -2.070 & 0.013 & $2.21 \times 10^{-5}$ & $1.49 \times 10^{1}$ & $7.01 \times 10^{3}$ & 3.4 & $-3.89 \times 10^{1}$ \\
\hline NGC 5024 & 4.17 & 6.47 & -1.995 & 0.071 & $1.44 \times 10^{-4}$ & $9.32 \times 10^{1}$ & $6.71 \times 10^{3}$ & 3.6 & $3.2 \times 10^{1}$ \\
\hline NGC 5053 & 0.73 & 1.35 & -2.450 & 0.041 & $2.91 \times 10^{-5}$ & $3.91 \times 10^{0}$ & $1.39 \times 10^{3}$ & 3.4 & $-5.56 \times 10^{-1}$ \\
\hline NGC 5139 & 33.40 & 53.55 & -1.647 & 0.271 & $1.3 \times 10^{-3}$ & $6.96 \times 10^{3}$ & $5.55 \times 10^{4}$ & 5.9 & $5.83 \times 10^{3}$ \\
\hline NGC 5272 & 3.60 & 5.95 & -1.391 & 0.097 & $7.93 \times 10^{-4}$ & $4.72 \times 10^{2}$ & $6.17 \times 10^{3}$ & 4.8 & $2.46 \times 10^{2}$ \\
\hline NGC 5286 & 3.59 & 8.51 & -1.727 & 0.103 & $3.89 \times 10^{-4}$ & $3.31 \times 10^{2}$ & $8.82 \times 10^{3}$ & 4.0 & $1.82 \times 10^{2}$ \\
\hline NGC 5466 & 0.55 & 1.09 & -1.865 & 0.075 & $2.05 \times 10^{-4}$ & $2.23 \times 10^{1}$ & $1.13 \times 10^{3}$ & 3.6 & $8.44 \times 10^{0}$ \\
\hline NGC 5634 & 2.20 & 3.98 & -1.869 & 0.081 & $2.2 \times 10^{-4}$ & $8.74 \times 10^{1}$ & $4.12 \times 10^{3}$ & 3.7 & $3.72 \times 10^{1}$ \\
\hline NGC 5694 & 3.67 & 6.13 & -2.017 & 0.046 & $8.84 \times 10^{-5}$ & $5.42 \times 10^{1}$ & $6.35 \times 10^{3}$ & 3.5 & $-8.95 \times 10^{-1}$ \\
\hline NGC 5824 & 8.49 & 12.58 & -2.174 & 0.058 & $7.78 \times 10^{-5}$ & $9.78 \times 10^{1}$ & $1.30 \times 10^{4}$ & 3.5 & $1.90 \times 10^{1}$ \\
\hline NGC 5904 & 3.68 & 6.55 & -1.259 & 0.041 & $4.51 \times 10^{-4}$ & $2.96 \times 10^{2}$ & $6.79 \times 10^{3}$ & 4.1 & $-4.10 \times 10^{1}$ \\
\hline NGC 5986 & 3.31 & 11.19 & -1.527 & 0.061 & $3.63 \times 10^{-4}$ & $4.06 \times 10^{2}$ & $1.16 \times 10^{4}$ & 3.9 & $9.56 \times 10^{1}$ \\
\hline NGC 6093 & 2.64 & 17.90 & -1.789 & 0.014 & $4.54 \times 10^{-5}$ & $8.13 \times 10^{1}$ & $1.86 \times 10^{4}$ & 3.5 & $-1.91 \times 10^{2}$ \\
\hline NGC 6121 & 0.93 & 7.53 & -1.166 & 0.050 & $6.82 \times 10^{-4}$ & $5.14 \times 10^{2}$ & $7.81 \times 10^{3}$ & 4.5 & $3.45 \times 10^{1}$ \\
\hline NGC 6139 & 3.48 & 8.66 & -1.593 & 0.033 & $1.68 \times 10^{-4}$ & $1.46 \times 10^{2}$ & $8.98 \times 10^{3}$ & 3.6 & $-6.08 \times 10^{1}$ \\
\hline NGC 6171 & 0.81 & 4.50 & -0.949 & 0.047 & $1.06 \times 10^{-3}$ & $4.76 \times 10^{2}$ & $4.67 \times 10^{3}$ & 5.4 & $3.78 \times 10^{0}$ \\
\hline NGC 6205 & 4.69 & 9.58 & -1.443 & 0.101 & $7.33 \times 10^{-4}$ & $7.02 \times 10^{2}$ & $9.92 \times 10^{3}$ & 4.6 & $3.8 \times 10^{2}$ \\
\hline NGC 6218 & 0.87 & 2.84 & -1.315 & 0.029 & $2.80 \times 10^{-4}$ & $7.96 \times 10^{1}$ & $2.94 \times 10^{3}$ & 3.8 & $-4.87 \times 10^{1}$ \\
\hline NGC 6229 & 2.88 & 5.78 & -1.129 & 0.044 & $6.54 \times 10^{-4}$ & $3.78 \times 10^{2}$ & $5.99 \times 10^{3}$ & 4.5 & $-2.28 \times 10^{1}$ \\
\hline NGC 6254 & 1.89 & 4.90 & -1.559 & 0.049 & $2.71 \times 10^{-4}$ & $1.32 \times 10^{2}$ & $5.08 \times 10^{3}$ & 3.8 & $6.24 \times 10^{0}$ \\
\hline NGC 6266 & 6.90 & 17.29 & -1.075 & 0.041 & $6.89 \times 10^{-4}$ & $1.19 \times 10^{3}$ & $1.79 \times 10^{4}$ & 4.6 & $-1.65 \times 10^{2}$ \\
\hline NGC 6273 & 6.57 & 14.18 & -1.612 & 0.161 & $8.03 \times 10^{-4}$ & $1.14 \times 10^{3}$ & $1.47 \times 10^{4}$ & 4.8 & $8.15 \times 10^{2}$ \\
\hline NGC 6341 & 3.12 & 8.43 & -2.239 & 0.083 & $9.61 \times 10^{-5}$ & $8.1 \times 10^{1}$ & $8.73 \times 10^{3}$ & 3.5 & $3.55 \times 10^{1}$ \\
\hline NGC 6362 & 1.16 & 3.21 & -1.092 & 0.017 & $2.75 \times 10^{-4}$ & $8.82 \times 10^{1}$ & $3.33 \times 10^{3}$ & 3.8 & $-1.54 \times 10^{2}$ \\
\hline NGC 6366 & 0.43 & 2.25 & -0.555 & 0.071 & $3.97 \times 10^{-3}$ & $8.92 \times 10^{2}$ & $2.33 \times 10^{3}$ & 13.3 & $3.08 \times 10^{2}$ \\
\hline NGC 6388 & 11.30 & 21.88 & -0.428 & 0.054 & $4.03 \times 10^{-3}$ & $8.82 \times 10^{3}$ & $2.27 \times 10^{4}$ & 13.5 & $1.22 \times 10^{3}$ \\
\hline NGC 6397 & 0.90 & 2.62 & -1.994 & 0.028 & $5.67 \times 10^{-5}$ & $1.49 \times 10^{1}$ & $2.72 \times 10^{3}$ & 3.5 & $-9.98 \times 10^{0}$ \\
\hline NGC 6402 & 7.39 & 19.12 & -1.130 & 0.053 & $7.86 \times 10^{-4}$ & $1.50 \times 10^{3}$ & $1.98 \times 10^{4}$ & 4.8 & $1.81 \times 10^{2}$ \\
\hline NGC 6441 & 12.50 & 24.24 & -0.334 & 0.079 & $7.35 \times 10^{-3}$ & $1.78 \times 10^{4}$ & $2.51 \times 10^{4}$ & 25.6 & $7.35 \times 10^{3}$ \\
\hline NGC 6535 & 0.13 & 3.65 & -1.963 & 0.035 & $7.61 \times 10^{-5}$ & $2.78 \times 10^{1}$ & $3.78 \times 10^{3}$ & 3.5 & $-9.35 \times 10^{0}$ \\
\hline NGC 6553 & 4.45 & 11.23 & -0.151 & 0.047 & $6.64 \times 10^{-3}$ & $7.45 \times 10^{3}$ & $1.16 \times 10^{4}$ & 22.6 & $7.45 \times 10^{1}$ \\
\hline NGC 6569 & 2.28 & 6.09 & -0.867 & 0.055 & $1.49 \times 10^{-3}$ & $9.11 \times 10^{2}$ & $6.31 \times 10^{3}$ & 6.4 & $1.39 \times 10^{2}$ \\
\hline NGC 6626 & 2.84 & 12.60 & -1.287 & 0.075 & $7.77 \times 10^{-4}$ & $9.79 \times 10^{2}$ & $1.31 \times 10^{4}$ & 4.7 & $3.72 \times 10^{2}$ \\
\hline NGC 6656 & 4.09 & 7.39 & -1.803 & 0.132 & $4.21 \times 10^{-4}$ & $3.11 \times 10^{2}$ & $7.66 \times 10^{3}$ & 4.0 & $2.02 \times 10^{2}$ \\
\hline NGC 6681 & 1.20 & 6.26 & -1.633 & 0.028 & $1.30 \times 10^{-4}$ & $8.14 \times 10^{1}$ & $6.48 \times 10^{3}$ & 3.5 & $-5.46 \times 10^{1}$ \\
\hline NGC 6715 & 16.20 & 23.68 & -1.559 & 0.183 & $1.04 \times 10^{-3}$ & $2.46 \times 10^{3}$ & $2.45 \times 10^{4}$ & 5.3 & $1.85 \times 10^{3}$ \\
\hline NGC 6752 & 2.32 & 4.81 & -1.583 & 0.034 & $1.77 \times 10^{-4}$ & $8.54 \times 10^{1}$ & $4.99 \times 10^{3}$ & 3.6 & $-3.20 \times 10^{1}$ \\
\hline NGC 6809 & 1.88 & 5.19 & -1.934 & 0.045 & $1.05 \times 10^{-4}$ & $5.44 \times 10^{1}$ & $5.38 \times 10^{3}$ & 3.5 & $-2.12 \times 10^{0}$ \\
\hline NGC 6838 & 0.63 & 1.64 & -0.736 & 0.039 & $1.43 \times 10^{-3}$ & $2.35 \times 10^{2}$ & $1.70 \times 10^{3}$ & 6.2 & $-4.60 \times 10^{1}$ \\
\hline NGC 6864 & 4.10 & 8.05 & -1.164 & 0.059 & $8.1 \times 10^{-4}$ & $6.51 \times 10^{2}$ & $8.34 \times 10^{3}$ & 4.8 & $1.37 \times 10^{2}$ \\
\hline NGC 7078 & 4.99 & 8.50 & -2.287 & 0.053 & $5.48 \times 10^{-5}$ & $4.65 \times 10^{1}$ & $8.80 \times 10^{3}$ & 3.5 & $5.47 \times 10^{0}$ \\
\hline NGC 7089 & 5.20 & 15.58 & -1.399 & 0.021 & $1.67 \times 10^{-4}$ & $2.61 \times 10^{2}$ & $1.61 \times 10^{4}$ & 3.6 & $-3.2 \times 10^{2}$ \\
\hline NGC 7099 & 1.39 & 4.30 & -2.356 & 0.037 & $3.26 \times 10^{-5}$ & $1.4 \times 10^{1}$ & $4.45 \times 10^{3}$ & 3.5 & $-3.72 \times 10^{0}$ \\
\hline Terzan 1 & 2.72 & 30.29 & -1.263 & 0.037 & $4.03 \times 10^{-4}$ & $1.22 \times 10^{3}$ & $3.14 \times 10^{4}$ & 4.0 & $-3.20 \times 10^{2}$ \\
\hline Terzan 5 & 7.60 & 17.56 & -0.092 & 0.295 & $5.14 \times 10^{-2}$ & $9.02 \times 10^{4}$ & $1.82 \times 10^{4}$ & - & $7.70 \times 10^{4}$ \\
\hline Terzan 8 & 0.58 & 25.47 & -2.255 & 0.098 & $1.1 \times 10^{-4}$ & $2.79 \times 10^{2}$ & $2.64 \times 10^{4}$ & 3.5 & $1.47 \times 10^{2}$ \\
\hline
\end{tabular}

Fig. 2 shows $t_{\text {end }}^{\mathrm{SF}}$ over $M_{\mathrm{ini}}$. As we can see, the majority of GCs takes around $4 \mathrm{Myr}$ until SF ends and $T_{\mathrm{SF}}$ is similar for most of them. According to the data we used from Yan et al. (2019) a $120 M_{\odot}$ star, which is the upper limit we used for the IMF, explodes after about 3.5 Myr. Therefore, in most
GCs SF stops soon after the first SNe explode. The implied dependency from Fig. 1 between $n_{\mathrm{SN}}$ and $[\mathrm{Fe} / \mathrm{H}]$ leads to a similar dependency between $t_{\mathrm{SF}}^{\text {end }}$ and $[\mathrm{Fe} / \mathrm{H}]$. After correcting for the effect of measurement errors (see Sec. A) SF stops for all GCs except for NGC 6441 after a similar time. 


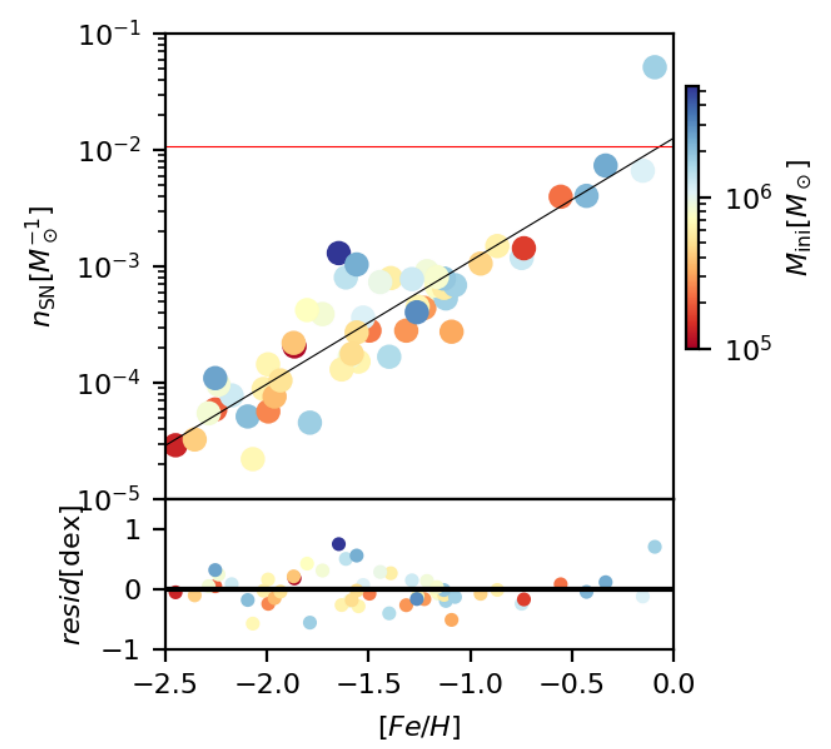

Figure 1. A visualization of the data in Table 1. The number of SNe per mass is plotted over the metallicity, colourcoded for the initial mass for all 55 GCs. The red line shows the maximum number of SN per mass possible for the canonical IMF.

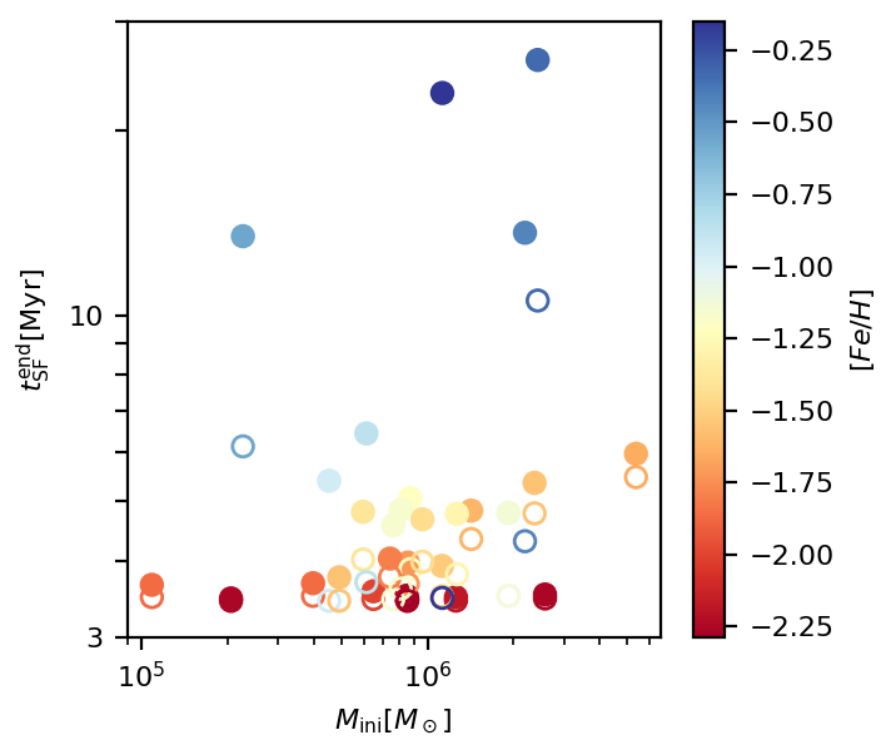

Figure 2. A visualization of the data in Table 1. The time required for the GCs until SF ends over their initial mass, colourcoded for the metallicity. The filled dots are the values from Table 1, the empty dots show the time when SF ends for the corrected numbers of SNe, $N_{\mathrm{SN}}^{\mathrm{subt}}$.

\section{DISCUSSION}

\subsection{Comparison with the expected number of $\mathrm{SNe}$}

As we can see in Table 1 the expected number of SNe, $N_{\mathrm{SN}}^{\mathrm{exp}}$, computed for the canonical IMF, is larger (often by one or two orders of magnitude) than the number of $\mathrm{SNe}, N_{\mathrm{SN}}$, as computed here, with the exception of Terzan 5 . This indi- cates that SF ends long before most of the SNe explode. At the same time, however, the effects neglected in our simplified analytical model may also play a role. These include a loss of SN ejecta due to their high velocities during GC formation (Renzini et al. 2015) and the fact that a portion of the most massive stars is not expected to turn into SNe but become failed SNe instead (Pejcha \& Thompson 2015; Sukhbold et al. 2016; Basinger et al. 2020; Neustadt et al. 2021). The fraction of those failed SNe is still unclear, however, the upper limit is estimated to be at around $50 \%$ (Lien et al. 2010; O'Connor \& Ott 2011; Horiuchi et al. 2014; Pejcha \& Thompson 2015; Sukhbold et al. 2016) and is, therefore, not sufficient to explain the difference between required and expected numbers of SNe in most GCs. Another effect that would reduce the number of SNe happening in a GC is the ejection of $\mathrm{O}$ stars due to stellar-dynamical encounters. However, Oh et al. (2015) found that only about $10 \%$ of O stars would be lost this way for GCs. One of the most important unknowns, when it comes to determining the number of SNe expected, is the exact shape of the IMF, since it determines the fraction of stars massive enough to turn into SNe. While we used the canonical IMF (Kroupa 2001; Kroupa et al. 2013) in this work, several authors found that the shape of the IMF varies depending on the GC parameters (Prantzos \& Charbonnel 2006; Marks et al. 2012; Bekki et al. 2017; Jeřábková et al. 2017; Kalari et al. 2018; Schneider et al. 2018; Chon et al. 2021). Additionally, the upper limit of the IMF can vary depending on the SF rate (e.g. Kroupa et al. 2013; Bekki et al. 2017; Stephens et al. 2017; Yan et al. 2017).

The larger $N_{\mathrm{SN}}$ for Terzan 5 might be a result of incomplete mixing (see Sec. 4.2) which can cause us to highly overestimate $N_{\mathrm{SN}}$ and $n_{\mathrm{SN}}$. It has also been suggested that Terzan 5 , similar to $\omega$ Cen, is not a usual GC, but the remnant of a larger structure (Massari et al. 2014).

The processes described here do not exclude each other, and it is likely that a multitude of the processes described here worked together to form the GCs we observe today.

The time when SF ends varies between 3.5 and 33.5 Myr with the majorities of cases clustering around $4 \mathrm{Myr}$. Since this result relies on the expected number of $\mathrm{SNe}$ it is also likely to be dependent on the upper end of the IMF. Variations in the iron output of $\mathrm{SNe}$ with different progenitor masses also play a role. If more massive stars (above $m_{\text {last }}$ ) would for example turn out to release twice as much iron as less massive ones only half of the stars would have to explode before the SF ends. Studies investigating the dependence of SN yields on mass and metallicity are yet to be performed.

In this paper we assumed that gas mixing and SF happen instantaneously after the $N_{\mathrm{SN}}$ th $\mathrm{SN}$ explodes. However, those processes are expected to take some time. Hydrodynamical simulations would be required to investigate the time required for mixing and the fractions of iron in the newly forming stars due to possible incomplete mixing. These simulations, are however, difficult and expensive to do. They would have to take different conditions like the density profile and the presence of dust into account and might even need to include radiative transfer. This makes the more detailed simulations more challenging and emphasises the usefulness of the method presented here. 


\subsection{The limitations of our method}

The main advantage of our method is that it is purely analytical. Due to the complexity of the problem hydrodynamical simulations would have to be very detailed, containing a number of effects like SN winds from different SNe reducing or enhancing each other. Therefore, they would be computationally very expensive to perform. However, it is important to pay close attention to the assumptions used in our model.

As discussed in Sec. $2.1 n_{\mathrm{SN}}$ scales approximately linearly with $\sigma_{[\mathrm{Fe} / \mathrm{H}]}$. This means that if we used only half the spread for our estimate we would also only get half the $n_{\mathrm{SN}}$. Therefore, an over- or underestimation of $\sigma_{[\mathrm{Fe} / \mathrm{H}]}$ will influence $n_{\mathrm{SN}}$ linearly, too. For a more detailed discussion on the effect of these errors see App. A.

As mentioned before, we are using the mean value of $[\mathrm{Fe} / \mathrm{H}]$ in our model and assume that it is exactly in the middle of the metallicities of both SN-enriched and not enriched populations. This means that if the second generation is comprised of more stars than the first one, we overestimate the number of SNe required and vice versa. It is important to note that this depends on the fraction of stars from each population in the sample of stars measured and not in the entire GC. The selection of GC stars, therefore, has a strong influence on the result. Milone et al. (2017) found, based on chromosome maps, that the number fraction of preSN-population stars declined with GC mass. In that case we would expect larger $n_{S N}$ for the more massive GCs. This is not the case with our current data.

Similarly to populations being under- or overrepresented, entire populations might be excluded from the sample. For example Bailin (2019) took the data for NGC 7089 from Masseron et al. (2019) which according to the author (private communication) did not include stars from the anomalous giant branch discovered by Yong et al. (2014). This can lead to an underestimate of the iron spreads.

Next to the systematic error for $[\mathrm{Fe} / \mathrm{H}]$, which was neglected in the Bailin (2019) data, there are several assumptions in our model that could influence our results: We assume a constant iron output from all our SNe, but in reality, more massive ejecta are expected at larger stellar masses and metallicities (Marassi et al. 2019). However, the chemical composition and mass fraction of SN yields is not fully understood yet (Chan et al. 2020). Additionally, the high-mass end of the IMF was found to vary with GC density and metallicity (Marks et al. 2012). These variations of the IMF would affect the composition of SN producing stars and therefore the average iron yield of the SNe.

Furthermore, we assume well mixed gas, i.e., the first generation of stars forms out of a homogeneous gas cloud, then the SNe polute the gas. The gas mixes so that the next generation of stars forms out of a homogeneous gas cloud as well. We also assume that no gas is lost from the GC. It is however not clear how the gas mixes and there is evidence for gas being lost from GCs (Calura et al. 2015; D'Ercole et al. 2016; Chantereau et al. 2020). If the gas is not well mixed pockets of larger metallicity remain in the gas, which would lead to stars with higher iron content and therefore larger iron abundance spreads in the GC as a whole. For example if the $\mathrm{SNe}$ ejecta mix only with half of the gas left over from the first SF event, we would only need half of the SNe to explain the observed iron spreads. The same would be true, if half of the gas was ejected from the GC before enrichment through SNe. Due to mass segregation the heavier stars which evolve into $\mathrm{SNe}$ are concentrated in the centre of the GC. Therefore, the gas ejected by them would largely be retained, while blowing out the gas further away from the GC's centre. This was further investigated by Jiménez et al. (2021), who found that the amount of gas retained depends on the initial gas mass and the core radius of the $\mathrm{GC}$.

We also have to consider the error introduced by $M_{\text {ini }}$ since $N_{\mathrm{SN}}$ depends linearly on it. It does not affect $n_{\mathrm{SN}}$ since the $M_{\text {ini }}$ in $N_{\text {SN }}$ cancels with the $M_{\text {ini }}$ in the denominator. In our calculations we assumed the same mean stellar mass, $\langle m\rangle$, for all GCs. Marks et al. (2012) found, however, that the IMF varies with the metallicity of the GC. The $M_{\text {ini }}$ we calculate is roughly proportional to $\langle m\rangle$ and therefore, $N_{\mathrm{SN}}$ is roughly proportional to $\langle m\rangle$, too.

\subsection{Individual clusters}

In the following sections we discuss individual GCs for which the number of SNe required is so large that even if assuming a large error (see App. A) at least $10^{3} \mathrm{SNe}$ are required to produce the observed iron spread. We will discuss if iron spreads for these GCs have been observed in other studies as well and whether or not spreads in the light-element content of GC stars has been observed as well. Note that spreads in light elements can also be caused from pollution of the GC gas before the first SNe for example through stellar mergers (Wang et al. 2020).

\subsubsection{NGC 5139}

The most famous GC on our list is NGC 5139 or $\omega$ Cen. The iron spread in this GC has been confirmed via chromosom maps by Marino et al. (2019). They identify three distinctive populations with different mean iron spreads, but also internal spreads. They find that the iron-rich population is also rich in sodium and nitrogen compared to the iron-poor one, but poor in oxygen. Since NGC 5139 is an unusual GC (very large mass, retrograde orbit, e.g. Dinescu et al. 1999; Bellini et al. 2018) and it has been suggested that it might have developed from Galactic threshing (Bekki \& Freeman 2003). According to our calculations, star formation in NGC 5139 ends after 6 Myr with the lowest mass star whose SN contributes to the iron spread being $34 M_{\odot}$. As mentioned in Sec. 4.2, composition of SN ejecta depending on mass and metallicity of the progenitor star is not fully understood. Therefore, we cannot draw any conclusion what consequences the SN ejecta might have on the light element content.

\subsection{2 $N G C 6388$}

In addition to the measurements featured in Bailin (2019), iron spreads in NGC 6388 are also visible from CaT measurements (Husser et al. 2020). This method relies on the correlation between the equivalent width of $\mathrm{Ca}$ lines and $[\mathrm{Fe} / \mathrm{H}]$ as found by Armandroff \& Zinn (1988). Husser et al. (2020) find a difference of 0.22 dex between different populations of this GC although with large measurement errors. Carretta \& Bragaglia (2018) claim for this GC to have no iron spread. Variations in both oxygen and sodium have been detected for this 
GC. Carretta et al. (2007) and Carretta \& Bragaglia (2018) also investigated the GC for other elements and found $\mathrm{Na}-\mathrm{O}$ and $\mathrm{Mg}-\mathrm{Al}$ anticorrelations. They find an excess of $\alpha$-process elements compared to the surrounding field stars. However, if the iron measurements are correct we find that SF lasts very long in this GC (13.6 Myr), therefore we would expect large abundance spread for the lighter elements as well. A consequence of this long lasting SF is that the mass of the last star whose $\mathrm{SN}$ ejecta contribute is also lower $\left(16 M_{\odot}\right)$.

\subsubsection{NGC 6441}

Similar to NGC 6388, NGC 6441 was investigated by Husser et al. (2020) using CaT measurements, however, they could not confirm an iron spread due to large measurement uncertainties. Gratton et al. (2007), whose data was used to create our dataset in Bailin (2019), also state that the measured variation in iron does not exceed measurement errors. Therefore it is likely, that the apparent iron spread in NGC 6441 is only an artefact and more precise observations are needed to confirm whether or not an iron spread in NGC 6441 exists. If our data is correct, however, $\mathrm{SF}$ in this GC lasts even longer, than in the previous GCs (26 Myr) which leads to a minimum mass for stars contributing to the iron spread of only $10 M_{\odot}$ which is close to the lower limit of stars turning into SNe.

\subsubsection{NGC 6715}

Marino et al. (2019) used chromosome maps to identify three populations in NGC 6715 and found iron spreads both within and between the different populations. They also identified a spread in sodium, oxygen and magnesium. According to Marino et al. (2019) oxygen and sodium are anticorrelated. $\mathrm{SF}$ lasted only for 5.3 Myr in this GC and, therefore, the minimum mass for stars contributing to the iron spread is very large $\left(39 M_{\odot}\right)$.

\subsubsection{Terzan 5}

As described in Sec. 4.1 Terzan 5 is not an ordinary GCs and was suggested to be the remnant of a larger structure (Massari et al. 2014). The lack of an Al-O anticorrelation, as is often observed in GCs, further underlines the unusual status of Terzan 5 (Origlia et al. 2011). This might be related to the number of $N_{\mathrm{SN}}$ being is above the number of $N_{\mathrm{SN}}^{e x p}$.

\section{CONCLUSIONS}

We presented a new method to estimate the number of SNe required to reproduce the iron abundance spreads observed in GCs. Since it is an analytical method we do not need to run any computationally expensive hydrodynamical simulations. As observational data improves, this method can be used to yield more precise estimates and adjusted to develop a clearer understanding of what the metallicities of different stellar populations are.

Based on the values given in Bailin (2019) we find that multiple $\mathrm{SNe}$ (up to $10^{5}$ ) are required to obtain the iron abundance spreads in most GCs. We also demonstrate how to estimate the time SF ends based on these values. In all cases (except for Terzan 5) the number of SNe needed is significantly smaller than the number expected for a canonical stellar population and SF ends after a few Myr.

To obtain more reliable results more detailed models including iron yields depending on stellar mass and metallicity are required. Future work should also include hydrodynamical simulations to investigate the degree of mixing the gas in the GCs experiences before the second population of stars forms after the first SNe. The dependency on the GCs' IMFs of different GC parameters should also be taken into account. Additionally, better measurements of present day iron abundance spreads in GCs need to be done.

\section{ACKNOWLEDGEMENTS}

We thank František Dinnbier for our many useful discussions. The authors acknowledge support from the Grant Agency of the Czech Republic under grant number 20-21855S.

\section{DATA AVAILABILITY}

The data used here has been cited and is available in published form.

\section{REFERENCES}

André P., Di Francesco J., Ward-Thompson D., Inutsuka S. I., Pudritz R. E., Pineda J. E., 2014, in Beuther H., Klessen R. S., Dullemond C. P., Henning T., eds, Protostars and Planets VI. University of Arizona Press, Tucson, p. 27 (arXiv:1312.6232), doi:10.2458/azu uapress 9780816531240-ch002

Armandroff T. E., Zinn R., 1988, AJ, 96, 92

Asplund M., Grevesse N., Sauval A. J., Scott P., 2009, ARA\&A, 47, 481

Bailin J., 2018, ApJ, 863, 99

Bailin J., 2019, ApJS, 245, 5

Banerjee S., Kroupa P., 2018, Formation of Very Young Massive Clusters and Implications for Globular Clusters. Springer International Publishing AG, Cham, p. 143, doi:10.1007/978-3319-22801-3_6

Basinger C. M., Kochanek C. S., Adams S. M., Dai X., Stanek K. Z., 2020, arXiv e-prints, p. arXiv:2007.15658

Baumgardt H., Makino J., 2003, MNRAS, 340, 227

Baumgardt H., Kroupa P., Parmentier G., 2008, MNRAS, 384, 1231

Bekki K., Freeman K. C., 2003, MNRAS, 346, L11

Bekki K., Jeřábková T., Kroupa P., 2017, MNRAS, 471, 2242

Bellini A., et al., 2018, ApJ, 853, 86

Blitz L., 1979, ApJ, 231, L115

Brinkmann N., Banerjee S., Motwani B., Kroupa P., 2017, A\&A, 600, A49

Calura F., Few C. G., Romano D., D'Ercole A., 2015, ApJ, 814, L14

Carretta E., Bragaglia A., 2018, A\&A, 614, A109

Carretta E., et al., 2007, A\&A, 464, 967

Carretta E., et al., 2009a, A\&A, 505, 117

Carretta E., Bragaglia A., Gratton R., D’Orazi V., Lucatello S., 2009b, A\&A, 508, 695

Chan C., Müller B., Heger A., 2020, MNRAS, 495, 3751

Chantereau W., Biernacki P., Martig M., Bastian N., Salaris M., Teyssier R., 2020, MNRAS, 493, 1306

Chon S., Omukai K., Schneider R., 2021, arXiv e-prints, p. arXiv:2103.04997 
Cottrell P. L., Da Costa G. S., 1981, ApJ, 245, L79

D'Ercole A., D'Antona F., Vesperini E., 2016, MNRAS, 461, 4088 Dinescu D. I., Girard T. M., van Altena W. F., 1999, AJ, 117, 1792 Dotter A., et al., 2010, ApJ, 708, 698

Ekström S., et al., 2012, A\&A, 537, A146

Elmegreen B. G., 2017, ApJ, 836, 80

Ferraro F. R., et al., 2009, Nature, 462, 483

Gratton R. G., et al., 2007, A\&A, 464, 953

Gratton R. G., Carretta E., Bragaglia A., 2012, A\&A Rev., 20, 50

Gratton R., Bragaglia A., Carretta E., D'Orazi V., Lucatello S., Sollima A., 2019, A\&A Rev., 27, 8

Hilker M., Baumgardt H., Sollima A., Bellini A., 2019, Proceedings of the International Astronomical Union, 14, 451-454

Honma M., Sofue Y., 1997, PASJ, 49, 453

Horiuchi S., Nakamura K., Takiwaki T., Kotake K., Tanaka M., 2014, MNRAS, 445, L99

Husser T.-O., et al., 2020, A\&A, 635, A114

Jeřábková T., Kroupa P., Dabringhausen J., Hilker M., Bekki K., 2017, A\&A, 608, A53

Jiménez S., Tenorio-Tagle G., Silich S., 2021, MNRAS, 505, 4669

Kalari V. M., Carraro G., Evans C. J., Rubio M., 2018, ApJ, 857, 132

Krause M., Charbonnel C., Decressin T., Meynet G., Prantzos N., Diehl R., 2012, A\&A, 546, L5

Kroupa P., 2001, MNRAS, 322, 231

Kroupa P., Weidner C., Pflamm-Altenburg J., Thies I., Dabringhausen J., Marks M., Maschberger T., 2013, The Stellar and Sub-Stellar Initial Mass Function of Simple and Composite Populations. Springer Netherlands, p. 115, doi:10.1007/978-94007-5612-0 4

Lada C. J., Lad̄a E. A., 2003, ARA\&A, 41, 57

Lardo C., Mucciarelli A., Bastian N., 2016, MNRAS, 457, 51

Lien A., Fields B. D., Beacom J. F., 2010, Phys. Rev. D, 81, 083001

Maoz D., Graur O., 2017, ApJ, 848, 25

Marassi S., Schneider R., Limongi M., Chieffi A., Graziani L., Bianchi S., 2019, MNRAS, 484, 2587

Marino A. F., et al., 2018, ApJ, 859, 81

Marino A. F., et al., 2019, MNRAS, 487, 3815

Marks M., Kroupa P., Dabringhausen J., Pawlowski M. S., 2012, MNRAS, 422, 2246

Massari D., et al., 2014, ApJ, 795, 22

Masseron T., et al., 2019, A\&A, 622, A191

Megeath S. T., et al., 2016, AJ, 151, 5

Milone A. P., 2016, Mem. Soc. Astron. Italiana, 87, 303

Milone A. P., et al., 2015, ApJ, 808, 51

Milone A. P., et al., 2017, MNRAS, 464, 3636

Montecinos C., Villanova S., Muñoz C., Cortés C. C., 2021, MNRAS, 503, 4336

Mucciarelli A., Lapenna E., Massari D., Pancino E., Stetson P. B., Ferraro F. R., Lanzoni B., Lardo C., 2015, ApJ, 809, 128

Neustadt J. M. M., Kochanek C. S., Stanek K. Z., Basinger C. M., Jayasinghe T., Garling C. T., Adams S. M., Gerke J., 2021, arXiv e-prints, p. arXiv:2104.03318

O'Connor E., Ott C. D., 2011, ApJ, 730, 70

Oh S., Kroupa P., Pflamm-Altenburg J., 2015, ApJ, 805, 92

Origlia L., et al., 2011, ApJ, 726, L20

Pancino E., Ferraro F. R., Bellazzini M., Piotto G., Zoccali M., 2000, ApJ, 534, L83

Pejcha O., Thompson T. A., 2015, ApJ, 801, 90

Portinari L., Chiosi C., Bressan A., 1998, A\&A, 334, 505

Prantzos N., Charbonnel C., 2006, A\&A, 458, 135

Renzini A., et al., 2015, MNRAS, 454, 4197

Schneider F. R. N., et al., 2018, Science, 359, 69

Shustov B. M., Wiebe D. S., 2000, MNRAS, 319, 1047

Stephens I. W., et al., 2017, ApJ, 834, 94

Sukhbold T., Ertl T., Woosley S. E., Brown J. M., Janka H. T., 2016, ApJ, 821, 38

Umeda H., Nomoto K., 2003, Nature, 422, 871
Usher C., Brodie J. P., Forbes D. A., Romanowsky A. J., Strader J., Pfeffer J., Bastian N., 2019, MNRAS, 490, 491

Wang L., Kroupa P., Takahashi K., Jerabkova T., 2020, MNRAS, 491, 440

Willman B., Strader J., 2012, AJ, 144, 76

Woosley S. E., Weaver T. A., 1995, ApJS, 101, 181

Yan Z., Jerabkova T., Kroupa P., 2017, A\&A, 607, A126

Yan Z., Jerabkova T., Kroupa P., Vazdekis A., 2019, A\&A, 629, A93

Yong D., et al., 2014, MNRAS, 441, 3396

\section{APPENDIX A: THE SENSITIVITY OF OUR MODEL TOWARDS MEASUREMENT ERRORS}

Our model is based on the Gaussian fit over the metallicity measurements of individual stars in the GCs. As such it is expected to be sensitive to errors of the individual metallicity measurements of those stars since they could stretch the Gaussian, making the iron spread look larger than it actually is. While Bailin (2019) argues that the random error in many of the previous studies have been overestimated (see his sec. 3.2 ), and therefore applied an independent random error estimation for each individual star using their Fe I lines, however, an underestimation of the random error of his method cannot be excluded based on the observational data available in the error analysis in Bailin's sec. 3.2. Indeed, it is unavoidable that some random error cannot be accounted for using the Fe I lines taken at the same time at the same spot on a CCD for a single star. Therefore, we investigate the effect of a larger random error on our result. We generated a population of synthetic GCs without any internal iron spread. Their metallicities ranged from $[\mathrm{Fe} / \mathrm{H}]=-2.5$ and -0.1 . We cre-

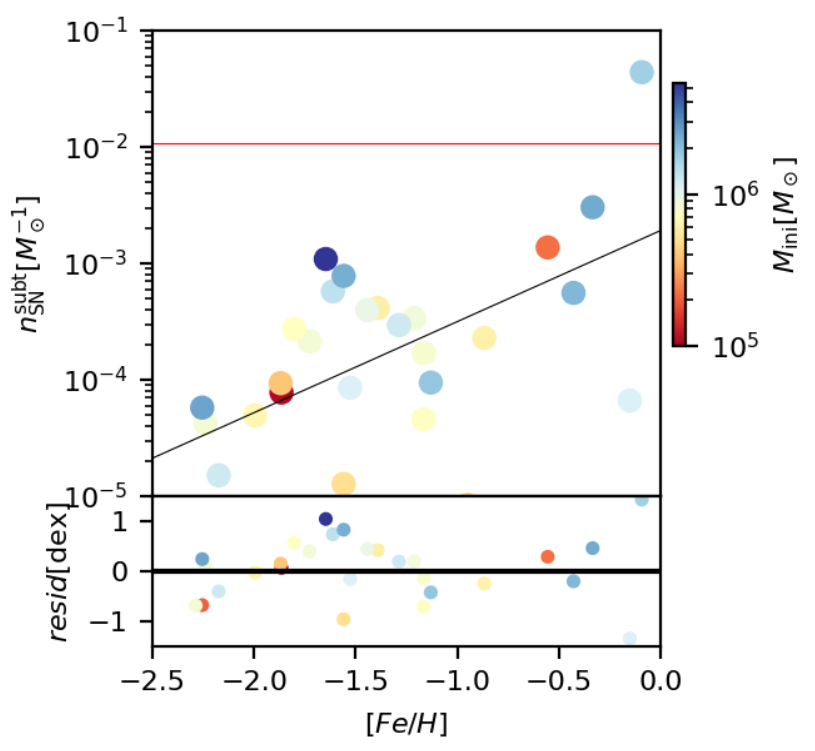

Figure A1. The same as Fig. 1 but with the error described in Sec. A substracted. Since approximately a third of our GCs end up with negative values for $N_{\mathrm{SN}}^{\text {subt }}$ only $26 \mathrm{GCs}$ are visible here. Three GCs are missing from the top panel because they have positive values below $10^{-5} M_{\odot}^{-1}$. Since the fit is done on an exponential scale and the residuals are in dex, only positive values were used here. 
ated a sample of 150 stars for each GC, which is consistent with the numbers of stars measured in the GCs with GIRAFFE (Carretta et al. 2009a). Since there is no significant dependency between the metallicity values and their error in Carretta et al. (2009a) we assigned their measurement errors to our stars randomly. We use the error values from Carretta et al. (2009a) because these measurements were also used by Bailin (2019), though Bailin (2019) argues that their error estimates are too large. Carretta et al. (2009a) computed those errors from the errors of the atmospheric parameters of the stars (effective temperatures and microturbulent velocity) and the equivalent widths. They multiplied these individual errors with the sensitivities of the abundance to variations of the corresponding parameter and then summed them by quadrature. For more information see Carretta et al. (2009a). The error values they computed this way follow a normal distribution. They varied between 0.003 dex and 0.287 dex with a mean of 0.130 dex.

The metallicity values of our synthetic stars were then shifted randomly within their error range, mimicking the effect of the error. We used Eqs. 2 and 4 to compute the apparent $n_{\mathrm{SN}}$ for the synthetic GCs. We then fitted $n_{\mathrm{SN}}$ over $[\mathrm{Fe} / \mathrm{H}]$ with the following function:

$n_{\mathrm{SN}}^{\mathrm{synt}}=a \times e^{b \times[\mathrm{Fe} / \mathrm{H}]}+c$

with $a, b$ and $c$ being free parameters and obtain $a=(1.3 \pm$ $0.1) \times 10^{-3} M_{\odot}^{-1}, b=2.3 \pm 0.1$ and $c=(0 \pm 2) \times 10^{-5} M_{\odot}^{-1}$. This shows that the error of our model increases sharply with $[\mathrm{Fe} / \mathrm{H}]$.

Assuming that the error values for all GCs are similar to those from Carretta et al. (2009a), we subtract this from $n_{\mathrm{SN}}$ and obtain the results in Table 1 with $n_{\mathrm{SN}}^{\text {subt }}=n_{\mathrm{SN}}-n_{\mathrm{SN}}^{\mathrm{synt}}$ and $N_{\mathrm{SN}}^{\mathrm{subt}}=n_{\mathrm{SN}}^{\mathrm{subt}} \times M_{\mathrm{ini}}$. As we can see $N_{\mathrm{SN}}^{\mathrm{subt}}$ is very close to zero or negative for a third of the GCs. However, for the majority of GCs a significant number of $\mathrm{SNe}$ is required to explain the measured iron spread even after correcting for the effect of the error of the metallicity measurements of individual stars.

Fig. A1 visualizes the results. As indicated by its definition $N_{\mathrm{SN}}^{\text {subt }}$ is smaller than $N_{\mathrm{SN}}$. The implied dependency is also less visible than before, however, it does not disappear. As discussed in Sec. 3 this also leads to smaller values of $t_{\mathrm{SF}}^{\text {end }}$. The larger residuals show that the implied dependency between $n_{\mathrm{SN}}$ and $[\mathrm{Fe} / \mathrm{H}]$ is weaker after the correction. The same is true for the Pearson coefficient. For the uncorrected values the Pearson coefficient is 0.91 and for the corrected values only 0.59 . This shows that at least in part the error in the individual measurements of the stars is responsible for the implied dependency.

This paper has been typeset from a $\mathrm{TE}_{\mathrm{E}} \mathrm{X} / \mathrm{L} \mathrm{T}_{\mathrm{E}} \mathrm{X}$ file prepared by the author. 